\title{
EDUCACIÓN A DISTANCIA VERSUS PRESENCIAL EN LA FORMACIÓN PEDAGÓGICA UNIVERSITARIA EN INGLATERRA. UN ESTUDIO COMPARADO.
}

\author{
DISTANCE LEARNING VERSUS FACE-TO-FACE EDUCATION IN \\ UNIVERSITY PEDAGOGICAL TRAINING IN ENGLAND. A COMPARATIVE \\ STUDY.
}

\author{
EDUCAÇÃO A DISTÂNCIA VERSUS PRESENCIAL NA FORMAÇÃO \\ PEDAGÓGICA UNIVERSITÁRIA DA INGLATERRA. UM ESTUDO \\ COMPARATIVO.
}

\author{
Alicia Sianes-Bautista \\ Universidad de Extremadura, España \\ https://orcid.org/0000-0003-2285-6937 \\ sianes@unex.es
}

Recibido: 08/03/2021 Revisado:14/04/2021 Aceptado:18/04/202 Publicado: 22/04/2021

Resumen: El estudio de la Pedagogía como disciplina en Inglaterra ha sido un ámbito de investigación que, hasta tiempos recientes, ha permanecido prácticamente inexplotado, incluso para los investigadores ingleses. Es una disciplina que, en este contexto, goza de una irrefutable juventud y en la que todavía es menester seguir investigando. El objetivo del presente trabajo consiste en contribuir a este ámbito de conocimiento mediante un análisis de las fortalezas y flaquezas de la formación pedagógica universitaria en Inglaterra. Empleando el método comparado se contrastan las titulaciones actuales de dos universidades prestigiosas: una a distancia (The Open University) y otra presencial (The University of Cambridge). Los resultados muestran que en todos los ciclos del EEES y en ambas universidades existen titulaciones pedagógicas que cubren las necesidades formativas y demandas del contexto inglés. Las principales diferencias halladas con respecto a factores como la flexibilidad, los contenidos, la excelencia académica y el prestigio universitario, entre otros, corresponden a disparidades acorde al ethos de las propias universidades, tanto presencial como a distancia. Se concluye que a partir de 1980 la Pedagogía se considera finalmente una disciplina académica en Inglaterra y, con el EEES se han solventado carencias formativas pedagógicas de antaño.

Palabras claves: Pedagogía; Educación Superior; Inglaterra; Educación Comparada; Educación a Distancia.

\begin{abstract}
The study of Pedagogy as a discipline in England has been a field of study that until recent times has remained practically unexplored even for English researchers. It is a discipline that, in this context, has an irrefutable youth, and in which it is still necesary further research. The objective of this work consists on contributing to this field of knowledge through an analysis of the strenghts and weakenesses of the university pedagogical training in England. Using the comparative methodology it contrasts the current degrees of two prestigious universities: a distance university (The Open University) and a face-to-face university (The University of Cambridge). Results show in all cycles of the EHEA and in both universities there are pedagogical degrees that cover the training needs and demands of the English context. The main found differences are in relation to aspects like flexibility, contents, the academic excellence and university prestige, among others, belong to disparities in accordance with the ethos of the universities themselves, both the face-to-face and the distance one. In conclussion it is from 1980
\end{abstract}


that Pedagogy has finally considered an academic discipline in England, and with the EHEA some pedagogical training lacks from yesteryear have been solved.

Keywords: Pedagogy; Higher Education; England; Comparative Education; Distance Education.

Resumo: O estudo da Pedagogia como disciplina na Inglaterra, e tudo relacionado a ela, tem sido uma área que até algumas décadas atrás apenas os próprios acadêmicos ingleses haviam investigado. É uma disciplina que, neste contexto, goza de uma juventude irrefutável e na qual é necessário continuar a pesquisar. O objetivo deste trabalho é contribuir para esse campo do conhecimento por meio de uma análise das potencialidades e fragilidades da formação pedagógica universitária na Inglaterra. Por meio do método comparativo, são contrastados os graus de uma instituição com metodologia a distância (The Open University) e outra presencial (The University of Cambridge). Os resultados mostram que nos três ciclos do EHEA e em ambas as universidades existem licenciaturas pedagógicas, contemplando as necessidades e exigências formativas do contexto inglês. As principais diferenças encontradas no que diz respeito a fatores como flexibilidade, conteúdo, busca de excelência acadêmica e prestígio universitário, entre outros, correspondem a disparidades de acordo com o ethos das próprias universidades, presencial e remotamente. Conclui-se que a partir de 1980, a Pedagogia passou a ser considerada uma disciplina acadêmica na Inglaterra e, com o EHEA, foram sanadas as deficiências formativas que outrora existiam.

Palavras-chave: Pedagogia; Educação superior; Inglaterra; Educação comparada; Educação a Distância.

Cómo citar este artículo: Sianes-Bautista, A. (2021). Educación a distancia versus presencial en la formación pedagógica universitaria en Inglaterra. Un estudio comparado. Hachetetepé. Revista cientifica en Educación y Comunicación, (22), 1-13. https://doi.org/10.25267/ Hachetetepe.2021.i22.1206

\section{INTRODUCCIÓN}

La Educación es un fenómeno consustancial al desarrollo de la persona, tan complejo que es preciso analizarlo desde multiplicidad de perspectivas para poder comprenderlo (Luengo Navas, 2002). El lenguaje es uno de los aspectos a los que hay que atender para comprender la falta de consenso en la construcción continental europea en relación con lo educativo, por ejemplo, el conflicto terminológico entre Pedagogía y Educación (Biesta, 2011). Mientras el ámbito anglosajón asume cierta unidad bajo el término Education, la heterogeneidad en la lengua alemana sale a relucir con conceptos como Bildung, Pädagogik o Erziehung, entre otros, o Pédagogie y Sciences de l'Éducation en la lengua francesa, etc. La temática que aquí concierne estudiar ha ido evolucionando a lo largo de los siglos de manera absolutamente disímil dentro del propio continente europeo (Judge, 1982), lo cual no únicamente se debe a la historia (Biesta, 2011), sino también a las propias tradiciones culturales que han ido condicionando el desarrollo de sus sistemas educativos (Pepin, 1998). Aunque durante los últimos años ha habido atisbos de convergencia entre algunos países en cuanto a la disciplina educativa, su concepción y denominación, todavía persisten diferencias significativas probablemente derivadas de su devenir histórico (Furlong y Whitty, 2017). Este hecho avala la heterogeneidad en tradiciones de la Educación y la Pedagogía como disciplina y su investigación tiene como finalidad, entre otras, la comprensión y el avance de los fenómenos y las realidades educativas. Asimismo, dado que las universidades son cada vez instituciones más competitivas e internacionalizadas, interesa conocer su 
posicionamiento acerca del valor que desde las mismas se le otorga al estudio de la Pedagogía y a su Investigación.

Furlong y Whitty (2017) clasifican el estudio de la Educación y la Pedagogía en tres agrupaciones que, si bien difieren considerablemente, no ha de cometerse el error de considerarlas inconexas pues, en ocasiones, podrían solaparse. El primer grupo, academic knowledge traditions o tradiciones de conocimiento académico, recoge las tradiciones de conocimiento pedagógico basadas en fundamentos de índole teórica. El segundo, practical knowledge traditions o tradiciones de conocimiento práctico, engloba las tradiciones pedagógicas enmarcadas en la praxis educativa. En otras palabras, serían aquellas que buscan la productividad por encima de la producción de conocimiento teórico. El tercero, integrated knowledge traditions o tradiciones de conocimiento integradas, sería en el que convergen las anteriores. Otro aspecto clave para el estudio de las tradiciones pedagógicas es el relativo a si su discurso es vertical u horizontal. El primero tiende a fundamentarse en el conocimiento académico mientras que, si pertenecen al discurso horizontal, predominará el conocimiento basado en la práctica (Hordern, 2017).

\section{METODOLOGÍA}

En la presente contribución se toma como prehipótesis la concepción de que la formación universitaria en Pedagogía en las universidades inglesas posee aspectos de mejora ligados al ethos de la disciplina, independientemente de si se imparte en universidades a distancia o presenciales. El objetivo del trabajo es transmitir las diferencias y similitudes entre la formación pedagógica en la enseñanza universitaria presencial y a distancia en Inglaterra. A tal efecto, se ha empleado el método comparado, propio de la Educación Comparada (Hernández Huerta, 2003), concretamente siguiendo los parámetros establecidos por García Garrido y García Ruíz (2012), quienes apuestan por un enfoque más postmodernista que positivista. A su juicio, las fases de un estudio comparado serían: la identificación del problema y emisión de una o varias prehipótesis; la delimitación del objeto; la delimitación del método; el estudio descriptivo; la formulación de la hipótesis comparativa; y el estudio comparativo. También se amplían sus horizontes ahondando en el transcurso histórico, el contexto y la filosofía que sustentan las convergencias y divergencias entre los países para dar respuesta a las demandas postmodernas propias de la actualidad (Egido Gálvez y Martínez-Usarralde, 2019) pues, tal y como sostienen en sus discursos comparatistas como Schriewer (2019), se antoja necesaria la reconciliación entre historia y comparación.

Dada la complejidad de analizar la totalidad de las universidades inglesas, se han tomado como unidades de comparación dos de las más prestigiosas y emblemáticas del territorio. En representación de las universidades presenciales estaría The University of Cambridge y The Open University representaría a las universidades a distancia. Esta última se ha seleccionado por ser la mayor universidad a distancia en Reino Unido y de toda Europa; mientras que The University of Cambridge se ha elegido por el internacionalmente reconocido prestigio que a ella se asocia y por conformar, además, una de las instituciones que constituyen el tradicional modelo universitario Oxbridge (Filmer, 1997) mundialmente conocido. Las fuentes primarias consultadas para extraer la información objeto de comparación han sido los planes de estudios de las titulaciones de las universidades, de acceso público en la página web de cada institución ${ }^{1}$. Para facilitar la consecución del objetivo general, se proponen tres específicos: a) divulgar el transcurso

e-ISSN:2172-7910

Doi:10.25267/ Hachetetepe.2021.i22.1206

Universidad de Cádiz 
y desarrollo histórico de la disciplina de la Pedagogía en Inglaterra, así como su ethos desde su origen hasta la actualidad; b) comparar las características y contenidos de las titulaciones pedagógicas de primer, segundo y tercer ciclo ofertadas por las universidades objeto de estudio; c) analizar sus bondades y desafíos, con el objeto de contribuir a su mejora y continuar incrementando su impacto y presencia en la Educación Superior de Inglaterra.

\section{PEDAGOGÍA COMO DISCIPLINA EN UNIVERSIDADES INGLESAS}

Las universidades escocesas fueron las primeras del Reino Unido en las que, ya en la primera mitad del siglo XX se ofertaban estudios de Pedagogía, aunque no se denominaban como tal, pues en los países anglosajones se inclinaban por el empleo de términos afines a Education, como Educational Studies, Educational Research y similares (Suasnábar, 2013). Un signo distintivo de estas titulaciones es que no tenían mucha vinculación con los centros o normal colleges destinados a la formación del profesorado. Éstos, hasta los años sesenta, reflejaban una clara influencia francesa tanto en principios como en metodologías, principalmente centradas en la más práctica dimensión de la educación (Furlong y Whitty, 2017; Hayhoe y Li, 2010). Esta separación institucional de Pedagogía y Formación del profesorado forma parte de la idiosincrasia pedagógica inglesa, pues ya el Cardenal Newman defendía que la formación docente podía ser impartida únicamente combinando educación liberal y experiencia práctica (Furlong y Whitty, 2017). Se pensaba que, fruto de esta separación, se incrementaría la puesta en práctica de técnicas ingeniosas e innovadoras procedentes de los centros y titulaciones de formación docente a la vez que surgirían cursos pedagógicos poco realistas, de escasa funcionalidad y aplicación práctica fundamentados en la dimensión teórica y académica de la educación (Crook, 2002), es decir, la Pedagogía.

Entre la década de los cuarenta y los cincuenta se produjo uno de los avances pedagógicos más significativos en Inglaterra al crearse y consolidarse la Standing Conference on Studies in Education (SCSE). Esta institución, bajo la dirección de Sir Fred Clarke, fue de las primeras en demandar la ausencia de reconocimiento de la Pedagogía como disciplina y destacó que en una sociedad de posguerra era esencial confiar en una mayor planificación educativa y delimitar objetivos claros (McCulloch, 2012). En 1952, fruto de la SCSE se crea el British Journal of Educational Studies con el propósito de enriquecer y propiciar la cultura de la investigación educativa. En este momento histórico reinaba una visión tan optimista de la educación que se contemplaba elevar su estatus hasta convertirla en disciplina. Desde que las universidades comenzaron a implicarse en la formación docente a finales del siglo XIX, han emergido diversas corrientes de pensamiento acerca del balance entre teoría y praxis en los cursos de formación del profesorado (Thomas, 1990). Sin embargo, al no existir en las universidades de entonces ninguna titulación de Educación o Pedagogía que no estuviera destinada a la formación docente, tradicionalmente la Educación Superior en Inglaterra no contaba con unos principios y fundamentos pedagógicos, en el más académico sentido (Tibble, 1966). Contrariamente, los departamentos e Institutes of Education ingleses han tendido a ser modestos y a contar con escaso personal, hecho que ha repercutido negativamente en su reputación en universidades y escuelas (Taylor, 1965).

En la literatura pedagógica inglesa tradicionalmente se confundían los conceptos de Pedagogía y Didáctica, hasta que la Pedagogía se fue haciendo cada vez más relevante a partir de la primera mitad del siglo XX (Schriewer, 2017). El Robbins Report (1963)

e-ISSN:2172-7910

Doi:10.25267/ Hachetetepe.2021.i22.1206

Universidad de Cádiz 
demandaba la importancia del desarrollo de la profesión docente en el Reino Unido y, con ello, la necesidad rediseñar los Training Colleges, convertidos en Colleges of Education (García Garrido, 2013). Además, se crea el Bachelor of Education, una titulación de cuatro cursos con aval universitario (Hilliard, 1969) en la que era menester equilibrar el conocimiento académico y el ejercicio profesional. Esto coadyuvó a la creación de un contexto propicio para dedicar más atención a los estudios pedagógicos, valorando su estructura, forma e importancia (Biesta, 2011). A esta la fueron sucediendo otras titulaciones de postgrado en las que ya se incluían contenidos introductorios sobre metodologías de investigación educativa (Crook, 2002) como los Advanced diplomas, Master in Education, Master of Arts in Education, que aportaban una sólida fundamentación para cursar estudios más avanzados de clara inclinación académica como Master of Philosophy y Philosophy Doctor. Este acercamiento académico a los estudios pedagógicos tuvo aún más aceptación cuando en 1966 se lanzó Students Library of Education, una serie de éxito sin precedentes en la que participaron académicos de renombre en las diferentes disciplinas.

A pesar de estos avances y los múltiples intentos por academizar los estudios de formación del profesorado entre los sesenta y los ochenta, la dimensión práctica ha predominado, probablemente a consecuencia de que ni el PGCE ni el Bachelor of Education pudieron cubrir tales carencias cognitivas con el rigor suficiente como para que se hubiera producido el desarrollo esperado (Kuhlee y Winch, 2017). Por lo tanto, hasta los ochenta Pedagogía y Ciencias de la Educación fueron posicionadas por los académicos británicos en un entorno de apoyo, a la vez que su conocimiento se erigía sobre pilares como la planificación, la formación y el asesoramiento educativo (Mares, 2014). Esta realidad hizo que, aun formando parte ya del mundo universitario, el estatus de la Pedagogía como disciplina fuera todavía considerablemente bajo (McCulloch, 2017). Progresivamente, entre la pérdida de interés por parte de los académicos en la formación del profesorado y la expansión de la Educación Superior, la década de los noventa ha sido crucial para el desarrollo de nuevas titulaciones pedagógicas en Inglaterra, convirtiéndose en un nuevo atractivo para sus universidades, a pesar de la reticencia y el escepticismo que todavía se arrastraba durante gran parte del siglo $\mathrm{XX}$ (Crook, 2002). Ya en el siglo XXI la Pedagogía ha alcanzado el nivel de disciplina académica, su estatus ha mejorado y se hace eco de su importancia para aspectos tales como la inclusión social, el Lifelong learning, el desarrollo de las habilidades de pensamiento en la infancia, etc. Junto a ello, en el White Paper Educational Excellence Everywhere se expone la necesidad de la investigación educativa para promover la buena praxis docente, entre otras cuestiones (Department for Education, 2016).

\section{RESULTADOS}

Los resultados muestran que Inglaterra es un país donde el desarrollo de la disciplina de la Pedagogía ha sido rápido y reciente, gozando de una irrefutable juventud. Este avance le ha permitido actualizarse a las demandas y requerimientos del Espacio Europeo de Educación Superior (en adelante, EEES) pues, actualmente, ambas universidades ofertan titulaciones pedagógicas en los tres ciclos que lo componen. A continuación - Tabla 1-, se muestra en una tabla comparativa en la que quedan recogidas las titulaciones pedagógicas halladas en ambas universidades, su duración y carga lectiva. Con anterioridad a analizar los datos, es menester comentar una particularidad de la carga lectiva en la Educación Superior en Inglaterra pues, aunque 
pertenecen al EEES, no es habitual que utilicen el European Credit Transfer and Accumulation System (ECTS), sino el Credit Accumulation and Transfer Scheme (CATS). Esto sucede en The Open University aunque, en universidades tan prestigiosas como The University of Cambridge, las titulaciones tampoco se ciñen al CATS, ya que esto no irá en detrimento de su excelencia, su posición ni su estatus en la academia. Ante tal peculiaridad, y para garantizar la homogeneidad en la comparación, la carga lectiva se expresa en horas y no en créditos. También se aprecia cómo todavía prevalece en las universidades inglesas el empleo de Education sobre Pedagogy que se comentaba al inicio, cuestión probablemente fruto de la escasa importancia que tradicionalmente ha tenido esta disciplina a lo largo de la historia en Inglaterra.

Tabla 1

Titulaciones universitarias de Pedagogía en The Open University y The University of Cambridge.

\begin{tabular}{|c|c|c|c|c|}
\hline Ciclo EEES & Universidad & Titulación & Cursos & Horas \\
\hline \multirow[t]{2}{*}{ Primer ciclo } & Open University & $\begin{array}{l}\text { Bachelor of Arts (Honours) in } \\
\text { Education studies - Primary }\end{array}$ & $3-6$ & 3.600 \\
\hline & $\begin{array}{l}\text { University of } \\
\text { Cambridge }\end{array}$ & $\begin{array}{l}\text { Undergraduate Advanced Diploma } \\
\text { Education, policy and international } \\
\text { development }\end{array}$ & 3 & 1.200 \\
\hline \multirow[t]{8}{*}{ Segundo ciclo } & Open University & $\begin{array}{l}\text { Postgraduate Certificate in } \\
\text { Professional Studies in Education }\end{array}$ & 1 & 600 \\
\hline & & $\begin{array}{l}\text { Postgraduate Diploma in Professional } \\
\text { Studies in Education }\end{array}$ & 2 & 1.200 \\
\hline & & $\begin{array}{l}\text { Master of Arts Online and Distance } \\
\text { Education }\end{array}$ & 2 & 1.800 \\
\hline & & Master degree in Education & $\begin{array}{c}3-6 \\
*_{\text {Fast track }}\end{array}$ & 1.800 \\
\hline & & Master of Philosophy (MPhil) & $1,5-2$ & - \\
\hline & $\begin{array}{l}\text { University of } \\
\text { Cambridge }\end{array}$ & $\begin{array}{l}\text { Master Education, Globalisation and } \\
\text { International Development }\end{array}$ & 1 & - \\
\hline & & $\begin{array}{l}\text { Master of Education (Educational } \\
\text { research) }\end{array}$ & $1-2$ & - \\
\hline & & Master of Philosophy (MPhil) & 1 & - \\
\hline \multirow[t]{4}{*}{ Tercer ciclo } & Open University & Doctorate of Phylosophy (Ph.D.) & $4-6$ & - \\
\hline & & Doctorate in Education (Ed.D.) & $\begin{array}{c}3 \\
* \text { Parcial }\end{array}$ & - \\
\hline & $\begin{array}{c}\text { University of } \\
\text { Cambridge }\end{array}$ & Doctorate of Phylosophy (Ph.D.) & $3-5$ & - \\
\hline & & Doctorate in Education (Ed.D.) & *Parcial & - \\
\hline
\end{tabular}

Fuente: Elaboración propia

\subsection{Comparación entre las titulaciones de primer ciclo}

A pesar de las diferencias terminológicas comentadas, es posible observar que en las titulaciones de primer ciclo también varía el tipo de titulación y la carga lectiva. La titulación Bachelor of Arts (Honours) in Education Studies se categoriza en los Honours 
Degrees, mientras que Education, Policy and International Development pertenece a la categoría de Undergraduate Advanced Diploma. Además, se deduce que la institución con modalidad a distancia (The Open University) ofrece una formación de primer ciclo más completa que la de la prestigiosa University of Cambridge, de modalidad presencial, pues la carga lectiva de la primera titulación triplica la de la segunda. Esta diferencia no perjudica a la homogeneidad de la comparación, pues ambas pertenecen al nivel $6 \mathrm{FHEQ}$, en otras palabras, son titulaciones de nivel equivalente.

A nivel de contenidos, conviene señalar la convergencia de ambas universidades ante la importancia otorgada a la política educativa en el curriculum de las titulaciones. Como principal diferencia está la inclinación hacia la praxis educativa palpable en la titulación de The Open University, frente a la tendencia predominantemente teórica de la titulación ofertada por The University of Cambridge, en la que claramente se perciben los propósitos de iniciar al alumnado en la investigación. Este es uno de los aspectos en los que más influye el ethos de cada universidad y sus criterios de admisión, pues el perfil del alumnado matriculado en las universidades a distancia suele ser más heterogéneo que el de las universidades presenciales. Además, The University of Cambridge posee unos criterios de admisión altamente exigentes, pues persiguen mantener su prestigio y excelencia académica. Al mismo tiempo, el prestigio de The Open University no depende únicamente de indicadores de excelencia, sino que otorga más importancia a factores como las posibilidades de acceso a la Universidad para el alumnado más heterogéneo, estudiantes no tradicionales, etc.

La flexibilidad es otra cuestión a tener en cuenta, pues puede abordarse desde la temporalización de las titulaciones o desde las posibilidades de especialización. En el primer caso, aunque ambas titulaciones pueden finalizarse en un mínimo de tres cursos académicos, en The Open University se facilita que el alumnado pueda duplicar el tiempo en el que termine los estudios. Por el contrario, la flexibilidad en The University of Cambridge no es temporal sino de especialización, es decir, ofrece una mayor posibilidad de combinar asignaturas optativas dentro de la misma titulación que en The Open University. Así, se garantiza una especialización mucho más personalizada.

\subsection{Comparación entre las titulaciones de segundo ciclo}

En las titulaciones de posgrado existe una mayor heterogeneidad en la oferta de titulaciones. La principal similitud hallada reside en que ambas instituciones ofrecen el Master of Philosophy (MPhil) con especialidad en Educación. En este nivel se perciben más diferencias entre la idiosincrasia de la universidad presencial y a distancia. Un ejemplo son las titulaciones ofertadas por The Open University (Postgraduate Certificate in Professional Studies in Education, Postgraduate Diploma in Professional Studies in Education Master of Arts in Online and Distance Education y Master degree in Education) cuya finalidad es indudablemente de especialización y naturaleza marcadamente profesional. Sin embargo, claramente se percibe la influencia del modelo universitario Oxbridge y la apuesta por titulaciones de naturaleza investigadora en The University of Cambridge (Master Education, Globalisation and International Development y Master of Education - Educational research). En este nivel The Open University es la institución que ofrece una mayor cantidad y variedad de titulaciones.

Con respecto a los contenidos, en el Master of Arts Online and Distance Education se forma en aspectos más teóricos de la Educación, es decir, los que en el presente trabajo se consideran pertenecientes a la disciplina pedagógica. En el Master of Arts Online and

e-ISSN:2172-7910

Doi:10.25267/ Hachetetepe.2021.i22.1206

Universidad de Cádiz 
Distance Education y Master Education, Globalisation and International Development, se busca formar al estudiantado en problemáticas educativas de actualidad, como la utilización de TIC en la educación, la globalización y su influjo en el ámbito educativo, los retos y desafíos emergentes del siglo XXI a los que deberán dar respuesta los Sistemas Educativos, etc. Estos temas se trabajan en el Master of Education desde la investigación educativa mediante la lectura de literatura científica y especializada, realizando investigaciones, desarrollando el espíritu crítico, poniendo en tela de juicio los resultados de investigaciones contemporáneas, etc. A pesar de la clara inclinación hacia la dimensión investigadora de la educación en la mayoría de las titulaciones, realmente la titulación académica por excelencia es el Master of Philosophy en Educación de ambas universidades. Ésta se destina a alumnado con intenciones de continuar su trayectoria académica e investigadora cursando posteriormente Doctorate of Philosophy ( $\mathrm{PhD})$.

En este nivel llama la atención que, a pesar de la clara inclinación profesional en la Pedagogía anglosajona, no sea posible realizar prácticas externas en ninguna titulación. Es decir, cuando en el curriculum del Master of Arts Online and Distance Education se contempla la formación y el desarrollo de habilidades cognitivas, prácticas y profesionales y en el curriculum de los Master of Education de ambas universidades se alude a las prácticas en investigación educativa, esto no implica que puedan realizarlas en centros de investigación o empresas. Igualmente, la especialización en las titulaciones de Postgraduate Certificate/Diploma in Professional Studies in Education hace referencia a la importancia de la adaptación docente a la práctica cotidiana, aunque no se especifica que realmente tengan oportunidad de practicar in situ, pues toda su metodología es online. Aunque esto pueda considerarse, a priori, una debilidad, se contrarresta con el rigor exigido en los diseños e investigaciones del alumnado. No solo han de ser viables y aplicables en la realidad que les rodea, sino que además deben aspirar a poder repercutir en las políticas y la concepción de la praxis educativa.

Las diferencias en duración y carga lectiva de las titulaciones de ambas universidades no pasan desapercibidas, cuestión que casi con total seguridad podría resolverse recurriendo a los elevados niveles de autonomía de las universidades en Inglaterra. Esta autonomía, lejos de limitar la investigación, avala la heterogeneidad y riqueza que reina en la Educación Superior inglesa. Se observa que la duración del Master degree in Education de The Open University es la única que puede reducirse, mediante una formación intensiva (fast track), mientras que la del Master of Philosophy (The Open University) y el Master of Education (University of Cambridge) puede ampliarse si la dedicación es parcial.

\subsection{Comparación entre las titulaciones de tercer ciclo}

El tercer ciclo es el que presenta mayor homogeneidad en las titulaciones de doctorado de ambas universidades y sus características. La formación de los Doctorate of Philosophy $(\mathrm{PhD})$ es claramente académica y persigue contribuir al avance de la ciencia mediante investigaciones rigurosas que den respuesta a problemáticas actuales, creen nuevas teorías educativas y contribuyan a la generación y difusión del conocimiento. Contrariamente, el Doctorate in Education (EdD) es un doctorado profesional a partir del cual se dota al estudiante de conocimientos para abordar en la práctica problemáticas actuales aplicando los avances científicos. Las principales diferencias en este nivel residen en la duración de los estudios. En el Doctorate of Philosophy de The Open University, la duración es ligeramente superior (1 curso) que en

e-ISSN:2172-7910

Doi:10.25267/ Hachetetepe.2021.i22.1206

Universidad de Cádiz 
su homónima en The University of Cambridge, tanto a tiempo parcial como completo. Ambas universidades coinciden en ofertar el Doctorate of Education a tiempo parcial y, en este caso, es The University of Cambridge la que cuenta con una duración dos cursos superior a la de la titulación en The Open University.

\section{DISCUSIÓN Y CONCLUSIONES}

Dando respuesta al primer objetivo específico de la investigación, consistente en divulgar el transcurso y desarrollo histórico de la disciplina de la Pedagogía en Inglaterra, así como su ethos desde su origen hasta la actualidad, se concluye que múltiples autores (Biesta, 2011; Furlong y Whitty, 2017; Lawn y Furlong, 2009) coinciden en que la investigación educativa en Inglaterra suele estar limitada, debido a su transcurso histórico y a su tradición educativa, a la praxis educativa y la formación del profesorado, por lo que el estatus académico de la Pedagogía ha sido considerablemente inferior al de otras disciplinas (Hoskin, 1993). Siguiendo los parámetros de Furlong y Whitty (2017) que se exponían al inicio, la Pedagogía como disciplina académica en las universidades de Inglaterra pertenecería a las practical knowledge traditions. Esta cuestión demuestra que el discurso pedagógico inglés coincide con la clasificación de discurso horizontal expuesto por Hordern (2017). Todo ello contribuye a que académicos como Alexander (2009) concibieran que el estudio de la Pedagogía en Inglaterra era una tarea ardua, manifestando que "es el más sorprendentemente prominente de los temas educativos que los comparatistas británicos han ignorado ${ }^{2} "$ (p. 923). Por consiguiente, investigar sobre Pedagogía en el ámbito inglés es fundamental pues, con ello, se coadyuva a construir el conocimiento científico pedagógico de una disciplina académica casi fantasma, debido a la escasez de publicaciones y estudios al respecto en Reino Unido (McCulloch y Cowan, 2017).

A lo largo del siglo XX, e incluso el XXI, ha habido autores que defendían el carácter interdisciplinar (Petrie, 1976; Simon, 1990) y multidisciplinar (McCulloch, 2017) de la Pedagogía. Estos son algunos ejemplos a partir de los cuales se mantenía que la Pedagogía no podía ser considerada una disciplina en sí misma, sino que necesitaba complementarse con otras afines, concepción dominante a lo largo de la segunda mitad del pasado siglo (McCulloch, 2002). Esta puede ser una de las explicaciones por las cuales ha sido complejo encontrar titulaciones con esencia y contenidos pedagógicos en las universidades analizadas. En general, es habitual que Education sea una especialización habilitante para el ejercicio docente de otra titulación. Se muestra coincidencia con Berry (2014), quien ampara que el profesorado precisa formación, no solo para ser conocedor de la importancia de su labor profesional a nivel pedagógico, sino también para ser profesionales críticos y reflexivos.

Según Mares (2014) no fue hasta 1980 cuando la academia británica concibió a la Pedagogía como ciencia de la Educación, como un escenario de apoyo sobre el cual centrar sus trabajos, ampliando sus contribuciones hacia temáticas como la planificación educativa, su capacitación y evaluación. En otras palabras, una disciplina que respondiera a las demandas educativas que Sir Fred Clarke ya consideraba necesarias desde los años cuarenta. La creciente preocupación por la Pedagogía se incrementa aún más con la publicación de 'Why no pedagogy in England'. En este trabajo, Simon (1981) demanda que en Inglaterra la Pedagogía distaba de ser coherente y sistemática, motivo por el que las y los educadores en Inglaterra carecían de formación en esa ciencia que, sin embargo, sí que se estudiaba en otros países centroeuropeos. Posteriormente, autores como Hoskin 
(1993) y Alexander (2004) se sumaban al aclamo de considerar la Pedagogía como una disciplina en sí misma. También con estas publicaciones se empieza a integrar más el uso de Pedagogy que, aunque todavía coexiste con Education y en ocasiones se siguen empleando como sinónimos, ya es más habitual que aparezca en manuales, publicaciones científicas, informes, etc. Se deduce que, probablemente gracias a avances como estos a finales del siglo XX en la concepción de la Pedagogía, el creciente interés por investigarla y la homogeneización de la Educación Superior en Europa con la creación del EEES, se ha abierto la posibilidad de crear y ofertar una mayor diversidad de titulaciones con esencia pedagógica en las universidades inglesas. De hecho, las titulaciones Postgraduate Certificate/Diploma in professional Education Studies son de las titulaciones pedagógicas más nuevas en The Open University, ofertadas por primera vez en el curso 2019/2020.

Dando respuesta al tercer objetivo específico, que buscaba analizar las bondades y desafíos de las titulaciones con el objeto de contribuir a su mejora y continuar incrementando su impacto y presencia en la Educación Superior de Inglaterra, cabe contemplar la particularidad de las titulaciones de doctorado. Es menester también aclarar que las apreciaciones aquí realizadas son generalistas y modestas, pues el debate sobre la coexistencia de titulaciones de doctorado profesional y académico se lleva estudiando desde la primera publicación al respecto en 1965 de Kerlinger. A él le han seguido múltiples estudios e investigaciones de considerable relevancia sobre esta temática (Andersen, 1983; Gregory, 1995; Kerlinger, 1965, 1968; Nelson y Coorough, 1994; Salomon, 2014; Shulman et al., 2006) en los que se abordaba el propósito de las titulaciones de doctorado en Educación, su nivel, si una titulación degrada a la otra, si el mero hecho de que coexistan $\mathrm{PhD}$ y EdD genera más confusión entre el mundo académico y el profesional, etc. Se puede concluir que las titulaciones de doctorado son, sin duda, una de las mayores bondades de las titulaciones pedagógicas en las universidades inglesas estudiadas. Esto es así tanto por la excelencia académica que persiguen, como por la sinigual profesionalización de las titulaciones de doctorado, tan tradicionalmente arraigadas a la exclusividad académica en múltiples naciones de todo el mundo.

Finalmente, dando respuesta al segundo objetivo específico, consistente en comparar las características y contenidos de las titulaciones pedagógicas de primer, segundo y tercer ciclo ofertadas por las universidades objeto de estudio; es posible concluir que las similitudes son bastantes al no observarse carencias de formación pedagógica en ningún ciclo del EEES en ninguna de las universidades. Es más, incluso hay titulaciones idénticas o muy parecidas que se ofertan en ambas. Las diferencias responden a factores como la flexibilidad en la duración de los estudios, que generalmente es mayor en The Open University que en The University of Cambridge; la búsqueda del prestigio universitario mediante la excelencia académica en The University of Cambridge frente al prestigio institucional a través de permitir el acceso y facilitar la finalización de estudios universitarios a personas con un perfil universitario no tradicional en The Open University; la inclinación hacia las titulaciones de investigación en The University of Cambridge contraria a la tendencia profesionalizante de las titulaciones de The Open University; entre otras. Estas diferencias responden más al ethos, la idiosincrasia y la seña de identidad de las propias universidades, más que al mero hecho de que su metodología sea a distancia o presencial. 


\section{NOTAS}

${ }^{1}$ http://www.open.ac.uk/ y https://www.cam.ac.uk/

${ }^{2}$ Traducción propia, en inglés el original: Pedagogy is the most startlingly prominent of the educational themes which British comparativists have ignored.

\section{REFERENCIAS BIBLIOGRÁFICAS}

Alexander, R. (2004). Still no pedagogy? Principle, pragmatism and compliance in primary education. Cambridge Journal of Education, 34(1), 7-33. http://doi.org/10.1080/0305764042000183106

Alexander, R. (2009). Towards a comparative pedagogy. En R. Cowen y A. M. Kazamias (Eds), International Handbook of Comparative Education (pp. 923-942). Springer.

Andersen, D. G. (1983). Differentiation of the $\mathrm{EdD}$ and $\mathrm{PhD}$ in Education. Journal of Teacher Education, 34(3), 55-58. http://doi.org/10.1177/002248718303400311

Biesta, G. (2011). Disciplines and Theory in the Academic Study of Education: A Comparative Analysis of the Anglo-American and Continental Construction of the Field. Pedagogy Culture and Society Culture \& Society, 2, 175-192. http://doi.org/10.1080/14681366.2011.582255

Crook, D. (2002). Educational Studies and Teacher Education. British Journal of Educational Studies, 50(1), 57-75. http://www.jstor.org/stable/3122405

Department for Education. (2016). White Paper Educational Excellence Everywhere. Department for Education. https://bit.ly/3gqdofK

Egido Gálvez, I., y Martínez Usarralde, M. J. (2019). La educación comparada, hoy. Síntesis.

Filmer, P. (1997). Disinterestedness and the Modern University. En A. Smith y F. Webster (Eds.), The Postmodern University? Contested Visions of Higher Education in Society (pp. 48-58). SRHE y Open University.

Furlong, J., \& Whitty, G. (2017). Knowledge traditions in the study of education. En G. Whitty y J. Furlong (Eds.), Knowledge and the study of Education: an international exploration (pp. 13-57). Symposium Books.

García Garrido, J. L. (2013). Sistemas educativos de hoy. Ediciones Académicas.

García Garrido, J. L. y García Ruíz, M. J. (2012). La metodología de la Educación comparada: del positivismo al postmodernismo. En J. L. García Garrido, M. J. García Ruíz y E. Gavari Starkie, La Educación Comparada en tiempos de Globalización (pp. 69-102). UNED.

Gregory, M. (1995). Implications of the Introduction of the Doctor of Education Degree in British Universities: can the $\mathrm{EdD}$ reach parts the $\mathrm{PhD}$ cannot? The vocational Aspect of Education, 47(2), 177-188. https://doi.org/10.1080/0305787950470206

Hayhoe, R., \& Li, J. (2010). The Idea of a Normal University in the 21st Century. Frontiers of Education in Education, 5(1), 74-103. https://doi.org/10.1007/s11516-010-0007-0

Hernández Huerta, J. L. (2003). Noah y Eckstein: hacia la cientificación de la educación comparada. Foro de Educación,1(1) 23-28. https://doi.org/10.14516/fde

Hilliard, F. (1969). Universities and the education of teachers. Educational Review, 21(2), 83-97. https://doi.org/10.1080/0013191690210201 
Hordern, J. (2017). Berstein's sociology of Knowledge and Education(al) Studies. En G. Whitty y J. Furlong (Eds.), Knowledge and the study of Education: an international exploration (pp. 191-210). Symposium Books.

Hoskin, K. (1993). Education and the Genesis of Disciplinary: the unexpected reversal. En E. Messer-Davidow, D. R. Shurrway y D. J. Sylvan (Eds.), Knowledges: Historical and critical studies in disciplinarity, (pp. 271-304). University Press of Virginia.

Judge, H. (1982). American Graduate Schools of Education: a view from abroad. Ford Foundation.

Kerlinger, F. N. (1965). The EdD and the PhD. Teachers College Record, 66(5), 434439. http://www.tcrecord.org/Content.asp?ContentId=2438

Kerlinger, F. N. (1968). The Doctoral Training of Research Specialists. Teachers College Record, 69(5), 477-483. http://www.tcrecord.org/Content.asp?ContentId=2006

Kuhlee, D., \& Winch, C. (2017). Teachers' knowledge in England and Germany: the conceptual background. En G. Whitty y J. Furlong (Eds.), Knowledge and the Study of Education: An International Exploration (pp. 231-254). Symposium Books.

Lawn, M., \& Furlong, J. (2009). The disciplines of education in the UK: Between the ghost and the shadow. Oxford Review of Education, 35(5), 541-552. https://doi.org/10.1080/03054980903216283

Luengo Navas, J. (2002). La Educación como objeto de conocimiento. El concepto de Educación. En M. M. del Pozo Andrés, J. L. Álvarez Castillo, J. Luengo Navas y E. Otero Urtza, Teorías e Instituciones contemporáneas de Educación (pp. 3047). Biblioteca Nueva. http://www.ugr.es/ fjjirios/pce/media/1EducacionConcepto.pdf

Mares, R. (2014). Redefining pedagogy in England nowadays. En I Boldea (Ed.), Identities in metamorphosis. Literature, discourse and multicultural dialogue. Secction: psychology and education sciences (pp. 176-181). Archipelag XXI Press.

McCulloch, G., \& Cowan, S. (2017). A Social History of Educational Studies and Research. Routledge.

McCulloch, G. (2002). Disciplines contributing to education? Educational studies and the disciplines. British Journal of Educational Studies 50(1), 100-119. https://doi.org/10.1111/1467-8527.t01-1-00193

McCulloch, G. (2012). The Standing Conference on Studies in Education - Sixty Years On. British Journal of Educational Studies, 60(4), 301-316. https://doi.org/10.1080/00071005.2012.691958

McCulloch, G. (2017). Education: an applied multidisciplinary field? En G. Whitty y J. Furlong (Eds), Knowledge and the Study of Education: An International Exploration (pp. 211-229). Symposium Books.

Nelson, J. K., \& Coorough, C. (1994). Content Analysis of the PhD versus EdD Dissertation. The Journal of Experimental Education, 62(2), 158-168. http://www.jstor.org/stable/20152407

Pepin, B. (1998, 17-20 septiembre). Curriculum, cultural traditions and pedagogy: understanding the work of teachers in England, France and Germany. [European Conference for Educational Research]. University of Ljubljana, Slovenia. https://bit.ly/3auZZPI 
Petrie, H. (1976). Do you see what I see? The Epistemology of Interdisciplinarity Inquiry. Educational Researcher, 5(2), 9-14. https://doi.org/10.3102/0013189X005002009

Salomon G. (2014). A Two-Track Doctorate in Education. En M. Ibarrola y L. W. Anderson (Eds.), The Nurturing of New Educational Researchers (pp. 65-72). Sense Publishers. https://doi.org/10.1007/978-94-6209-698-1_5

Schriewer, J. (2017). Between the Philosophy of Self-cultivation and Empirical Research: educational studies in Germany. En G. Whitty y J. Furlong (Eds), Knowledge and the Study of Education: An International Exploration (pp. 75-100). Symposium Books.

Schriewer, J. (2019). La reconciliación entre la historia y la comparación. Revista Española de Educación Comparada, 34, 148-162. https://doi.org/10.5944/reec.34.2019.25170

Shulman, S., Golde, C. M., Bueschel, A. C., \& Garabedian, K. J. (2006). Reclaiming Education's Doctorates: A critique and a proposal. Educational Researcher, 35(3), 25-32. https://doi.org/10.3102\%2F0013189X035003025

Simon, B. (1981). Why no pedagogy in England? En B. Simon y W. Taylor (Eds), Education in the eighties: the central issues (pp. 124-145). Batsford.

Simon, B. (1990). The Study of Education as a University Subject. En J. B. Thomas (Ed.), British Universities and Teacher Education: a century of change (pp. 125-142). The Falmer Press.

Suasnábar, C. (2013). La institucionalización de la educación como campo disciplinar. Un análisis desde la perspectiva de la historia social de las ciencias sociales. Revista Mexicana de Investigación Educativa, 59(18), 1281-1304. https://bit.ly/2P8yoMI

Taylor, W. (1965). The University Teacher of Education in England. Comparative Education, 1(3), 193-201. https://doi.org/10.1080/0305006650010306

Thomas, J. (1990). British Universities and Teacher Education. Falmer.

Tibble, J. W. (1966). The Study of Education. Routledge \& Keagan Paul. 